\title{
FURTHER LOG-SINE AND LOG-COSINE INTEGRALS
}

\author{
JunESANG ChOI*
}

\begin{abstract}
Motivated essentially by their potential for applications in a wide range of mathematical and physical problems, the log-sine and log-cosine integrals have been evaluated, in the existing literature on the subject, in many different ways. Very recently, Choi [6] presented explicit evaluations of some families of log-sine and log-cosine integrals by making use of the familiar Beta function. In the present sequel to the investigation [6], we evaluate the log-sine and log-cosine integrals involved in more complicated integrands than those in [6], by also using the Beta function.
\end{abstract}

\section{Introduction and preliminaries}

Motivated essentially by their potential for applications in a wide range of mathematical and physical problems, the log-sine and log-cosine integrals have been evaluated, in the existing literature on the subject, in many different ways (see, for example, $[2,3,6,7,9,10,13,15,17,18]$ and the references therein). By making use of the familiar Beta function $B(\alpha, \beta)$ (see, for example, [18, p. 8, Eq. (43)])

$$
B(\alpha, \beta)=\left\{\begin{array}{l}
\int_{0}^{1} t^{\alpha-1}(1-t)^{\beta-1} d t \quad(\Re(\alpha)>0 ; \Re(\beta)>0) \\
\frac{\Gamma(\alpha) \Gamma(\beta)}{\Gamma(\alpha+\beta)} \quad\left(\Re(\alpha)<0 ; \Re(\beta)<0 ; \alpha, \beta \notin \mathbb{Z}_{0}^{-}\right),
\end{array}\right.
$$

Received July 24, 2013; Accepted October 11, 2013.

2010 Mathematics Subject Classification: Primary 11M06, 11M35, 33B15; Secondary 11B68, 11B73, 11M36, 33B30.

Key words and phrases: Beta function, Gamma function, log-sine and log-cosine integrals, harmonic numbers, generalized harmonic numbers, odd harmonic numbers, generalized odd harmonic numbers, Riemann Zeta function, Hurwitz (or generalized) Zeta function, Psi(or Digamma) function, Polygamma functions, Euler-Mascheroni constant. 
where $\mathbb{Z}_{0}^{-}:=\mathbb{Z}^{-} \cup\{0\}, \mathbb{Z}^{-}:=\{-1,-2,-3, \ldots\}$, and $\Gamma$ is the familiar Gamma function, Choi [6] presented evaluations of some families of logsine and log-cosine integrals. In the present sequel to the investigation [6], we evaluate the log-sine and log-cosine integrals involved in more complicated integrands than those in [6], by also using the Beta function (1.1).

Setting $t=\sin ^{2} \theta$ in (1.1), we have the following equivalent form of the Beta function $B(\alpha, \beta)$ :

$$
B(\alpha, \beta)=2 \int_{0}^{\pi / 2}(\sin \theta)^{2 \alpha-1}(\cos \theta)^{2 \beta-1} d \theta \quad(\Re(\alpha)>0 ; \Re(\beta)>0) .
$$

For our purpose, let us replace $\alpha$ and $\beta$ by $\mu+1$ and $\nu+1$, respectively, in (1.2) and we get:

$$
\begin{aligned}
& \int_{0}^{\pi / 2}(\sin \theta)^{2 \mu+1}(\cos \theta)^{2 \nu+1} d \theta=\frac{\Gamma(\mu+1) \Gamma(\nu+1)}{2 \Gamma(\mu+\nu+2)} \\
&(\Re(\mu)>-1 ; \Re(\nu)>-1) .
\end{aligned}
$$

Differentiating each side of (1.3) $p$ times with respect to the variable $\mu$, we obtain

$$
\begin{aligned}
& \int_{0}^{\pi / 2}(\log \sin \theta)^{p}(\sin \theta)^{2 \mu+1}(\cos \theta)^{2 \nu+1} d \theta \\
&= \frac{1}{2^{p+1}} \frac{\partial^{p}}{\partial \mu^{p}}\left[\frac{\Gamma(\mu+1) \Gamma(\nu+1)}{\Gamma(\mu+\nu+2)}\right] \\
&\left(\Re(\mu)>-1 ; \Re(\nu)>-1 ; p \in \mathbb{N}_{0}:=\mathbb{N} \cup\{0\}\right),
\end{aligned}
$$

where $\mathbb{N}$ is the set of positive integers. Differentiating each side of (1.4) $q$ times with respect to the variable $\nu$, we get

$$
\begin{aligned}
\int_{0}^{\pi / 2} & (\log \sin \theta)^{p}(\log \cos \theta)^{q}(\sin \theta)^{2 \mu+1}(\cos \theta)^{2 \nu+1} d \theta \\
& =\frac{1}{2^{p+q+1}} \frac{\partial^{q}}{\partial \nu^{q}} \frac{\partial^{p}}{\partial \mu^{p}}\left[\frac{\Gamma(\mu+1) \Gamma(\nu+1)}{\Gamma(\mu+\nu+2)}\right] \\
& \left(\Re(\mu)>-1 ; \Re(\nu)>-1 ; p, q \in \mathbb{N}_{0}:=\mathbb{N} \cup\{0\}\right) .
\end{aligned}
$$

We also need the following notations and functions. The generalized harmonic numbers $H_{n}^{(s)}$ of order $s$ are defined by ( $c f$. [1]; see also [11], $[17$, p. 156] and [18, Section 3.5]) 


$$
H_{n}^{(s)}:=\sum_{j=1}^{n} \frac{1}{j^{s}} \quad(n \in \mathbb{N} ; s \in \mathbb{C})
$$

and

$$
H_{n}:=H_{n}^{(1)}=\sum_{j=1}^{n} \frac{1}{j} \quad(n \in \mathbb{N})
$$

are the harmonic numbers. Here $\mathbb{C}$ denotes the set of complex numbers, and we assume that

$$
H_{0}:=0, \quad H_{0}^{(s)}:=0 \quad(s \in \mathbb{C} \backslash\{0\}) \quad \text { and } \quad H_{0}^{(0)}:=1 .
$$

The generalized harmonic functions $H_{n}^{(s)}(z)$ are defined by (see $[4,11]$; see also $[14,16])$

$$
H_{n}^{(s)}(z):=\sum_{j=1}^{n} \frac{1}{(j+z)^{s}} \quad\left(n \in \mathbb{N} ; z \in \mathbb{C} \backslash \mathbb{Z}^{-} ; s \in \mathbb{C}\right),
$$

so that, obviously,

$$
H_{n}^{(s)}(0)=H_{n}^{(s)} .
$$

The generalized odd harmonic numbers $O_{n}^{(s)}$ of order $s$ are defined by

$$
O_{n}^{(s)}:=\sum_{j=1}^{n} \frac{1}{(2 j-1)^{s}} \quad(n \in \mathbb{N} ; s \in \mathbb{C})
$$

and

$$
O_{n}:=O_{n}^{(1)}=\sum_{j=1}^{n} \frac{1}{2 j-1} \quad(n \in \mathbb{N})
$$

are the odd harmonic numbers.

The Riemann Zeta function $\zeta(s)$ is defined by (see, for example, [18, Section 2.3])

$$
\zeta(s):= \begin{cases}\sum_{n=1}^{\infty} \frac{1}{n^{s}}=\frac{1}{1-2^{-s}} \sum_{n=1}^{\infty} \frac{1}{(2 n-1)^{s}} & (\Re(s)>1) \\ \frac{1}{1-2^{1-s}} \sum_{n=1}^{\infty} \frac{(-1)^{n-1}}{n^{s}} & (\Re(s)>0 ; s \neq 1),\end{cases}
$$


which is an obvious special case of the Hurwitz (or generalized) Zeta function $\zeta(s, a)$ defined by

$$
\zeta(s, a):=\sum_{k=0}^{\infty}(k+a)^{-s} \quad\left(\Re(s)>1 ; a \in \mathbb{C} \backslash \mathbb{Z}_{0}^{-}\right) .
$$

Equation (1.6) can be written in the following form:

$$
H_{n}^{(s)}=\zeta(s)-\zeta(s, n+1) \quad(\Re(s)>1 ; n \in \mathbb{N})
$$

by recalling the well-known (easily-derivable) relationship between the Riemann Zeta function $\zeta(s)$ and the Hurwitz (or generalized) Zeta function $\zeta(s, a)$ (see [17, Eq. 2.3(9)]):

$$
\zeta(s)=\zeta(s, n+1)+\sum_{k=1}^{n} k^{-s} \quad\left(n \in \mathbb{N}_{0}\right) .
$$

The Polygamma functions $\psi^{(n)}(s)(n \in \mathbb{N})$ are defined by

$$
\psi^{(n)}(s):=\frac{d^{n+1}}{d z^{n+1}} \log \Gamma(s)=\frac{d^{n}}{d s^{n}} \psi(s) \quad\left(n \in \mathbb{N}_{0} ; s \in \mathbb{C} \backslash \mathbb{Z}_{0}^{-}\right),
$$

where $\psi(s)$ denotes the Psi (or Digamma) function defined by

$$
\psi(s):=\frac{d}{d s} \log \Gamma(s) \quad \text { and } \quad \psi^{(0)}(s)=\psi(s) \quad\left(s \in \mathbb{C} \backslash \mathbb{Z}_{0}^{-}\right) .
$$

A well-known (and potentially useful) relationship between the Polygamma functions $\psi^{(n)}(s)$ and the generalized Zeta function $\zeta(s, a)$ is given by

$$
\begin{gathered}
\psi^{(n)}(s)=(-1)^{n+1} n ! \sum_{k=0}^{\infty} \frac{1}{(k+s)^{n+1}}=(-1)^{n+1} n ! \zeta(n+1, s) \\
\left(n \in \mathbb{N} ; s \in \mathbb{C} \backslash \mathbb{Z}_{0}^{-}\right) .
\end{gathered}
$$

It is also easy to have the following expression (cf. [17, Eq. 1.2(54)]):

(1.18) $\psi^{(m)}(s+n)-\psi^{(m)}(s)=(-1)^{m} m ! H_{n}^{(m+1)}(s-1) \quad\left(m, n \in \mathbb{N}_{0}\right)$,

which immediately gives $H_{n}^{(s)}$ the following another expression ( $c f$. [12, Eq. (20)]):

$$
H_{n}^{(m)}=\frac{(-1)^{m-1}}{(m-1) !}\left[\psi^{(m-1)}(n+1)-\psi^{(m-1)}(1)\right] \quad\left(m \in \mathbb{N} ; n \in \mathbb{N}_{0}\right) .
$$




\section{Some properties and special values of the Psi and Gamma functions}

In this section, for easy reference in the next section, we recall some properties and special values of the Psi and Gamma functions. The fundamental functional relation for the Gamma function is given as follows:

$$
\Gamma(z+1)=z \Gamma(z),
$$

so that, obviously,

$$
\begin{array}{cc}
\Gamma(z)=\frac{\Gamma(z+n)}{z(z+1) \cdots(z+n-1)} \quad\left(n \in \mathbb{N}_{0}\right) . \\
\psi(z+n)=\psi(z)+H_{n}(z-1) \quad\left(n \in \mathbb{N}_{0}\right),
\end{array}
$$

which is an obvious special case $(m=0)$ of (1.18).

$$
\begin{gathered}
\Gamma(n+1)=n ! \quad \text { and } \quad \Gamma\left(n+\frac{1}{2}\right)=\frac{(2 n) ! \sqrt{\pi}}{2^{2 n} n !} \quad\left(n \in \mathbb{N}_{0}\right) . \\
\psi(n)=-\gamma+H_{n-1} \quad(n \in \mathbb{N}),
\end{gathered}
$$

where $H_{0}:=0$ and $\gamma$ denotes the Euler-Mascheroni constant defined by

$$
\gamma:=\lim _{n \rightarrow \infty}\left(\sum_{k=1}^{n} \frac{1}{k}-\log n\right) \cong 0.577215664901532860606512 \cdots .
$$

$$
\psi\left(n+\frac{1}{2}\right)=-\gamma-2 \log 2+2 O_{n} \quad\left(n \in \mathbb{N}_{0}\right) .
$$

$$
\begin{gathered}
\psi\left(\frac{1}{2}\right)=-\gamma-2 \log 2 . \\
H_{n}^{(s)}\left(-\frac{1}{2}\right)=2^{s} O_{n}^{(s)} \quad(n \in \mathbb{N} ; s \in \mathbb{C}) . \\
\psi^{\prime}(n+1)-\psi^{\prime}\left(n+m+\frac{5}{2}\right) \\
=-\frac{\pi^{2}}{3}+4 O_{n+m+2}^{(2)}-H_{n}^{(2)} \quad\left(n, m \in \mathbb{N}_{0}\right) .
\end{gathered}
$$




$$
\begin{gathered}
\psi^{(2)}(n+1)-\psi^{(2)}\left(n+m+\frac{5}{2}\right) \\
=2\left(6 \zeta(3)+H_{n}^{(3)}+8 O_{n+m+2}^{(3)}\right) \quad\left(n, m \in \mathbb{N}_{0}\right) . \\
\psi^{(2)}\left(n+\frac{3}{2}\right)-\psi^{(2)}\left(n+m+\frac{5}{2}\right) \\
=-2 H_{m+1}^{(3)}\left(n+\frac{1}{2}\right) \quad\left(n, m \in \mathbb{N}_{0}\right) .
\end{gathered}
$$

\section{Log-Sine and its related integrals}

For convenience, let

$$
f(\mu, \nu):=\frac{\Gamma(\mu+1) \Gamma(\nu+1)}{\Gamma(\mu+\nu+2)} .
$$

Taking the logarithmic partial derivative of $f(\mu, \nu)$ with respect to the variable $\mu$, we get

$$
\frac{\partial}{\partial \mu} f(\mu, \nu)=f(\mu, \nu)[\psi(\mu+1)-\psi(\mu+\nu+2)] .
$$

Then, applying Leibniz's rule for differentiation to Equation (3.1), we obtain

$$
\begin{aligned}
\frac{\partial^{k+1}}{\partial \mu^{k+1}} f(\mu, \nu)= & \sum_{j=0}^{k}\left(\begin{array}{c}
k \\
j
\end{array}\right) \frac{\partial^{j}}{\partial \mu^{j}} f(\mu, \nu) \\
& \cdot \frac{\partial^{k-j}}{\partial \mu^{k-j}}[\psi(\mu+1)-\psi(\mu+\nu+2)] \quad\left(k \in \mathbb{N}_{0}\right) .
\end{aligned}
$$

Now, from Equations (1.4), (3.1) and (3.2), we find the following formula:

$$
\begin{aligned}
& \int_{0}^{\pi / 2}(\log \sin \theta)^{2}(\sin \theta)^{2 \mu+1}(\cos \theta)^{2 \nu+1} d \theta \\
&(3.3)= \frac{1}{8} \frac{\Gamma(\mu+1) \Gamma(\nu+1)}{\Gamma(\mu+\nu+2)} \\
& \cdot\left[(\psi(\mu+1)-\psi(\mu+\nu+2))^{2}+\psi^{\prime}(\mu+1)-\psi^{\prime}(\mu+\nu+2)\right] \\
&(\Re(\mu)>-1 ; \Re(\nu)>-1),
\end{aligned}
$$

where the prime' indicates the differentiation with respect to the variable $\mu$. 
Here, we present some interesting special cases of (3.3) without their proofs. It is noted that each of the formulas presented here can be easily derived by using suitably chosen identities and, if necessary, their easily-derivable variants, given in Sections 1 and 2 .

(1) $(\mu, \nu)=(n, m) \in \mathbb{N}_{0} \times \mathbb{N}_{0}$.

$$
\begin{aligned}
& \int_{0}^{\pi / 2}(\log \sin \theta)^{2}(\sin \theta)^{2 n+1}(\cos \theta)^{2 m+1} d \theta \\
& =\frac{n ! m !}{8(n+m+1) !}\left[\left(H_{n}-H_{n+m+1}\right)^{2}+H_{m+1}^{(2)}(n)\right] .
\end{aligned}
$$

(2) $(\mu, \nu)=(n, m+1 / 2)$ and $(n, m) \in \mathbb{N}_{0} \times \mathbb{N}_{0}$.

$$
\begin{aligned}
& \int_{0}^{\pi / 2}(\log \sin \theta)^{2}(\sin \theta)^{2 n+1}(\cos \theta)^{2(m+1)} d \theta \\
& =\frac{2^{2 n-1} n !(2 m+2) !(n+m+2) !}{(m+1) !(2 n+2 m+4) !} \\
& \quad \cdot\left[\left(2 \log 2+H_{n}-2 O_{n+m+2}\right)^{2}-\frac{\pi^{2}}{3}+4 O_{n+m+2}^{(2)}-H_{n}^{(2)}\right] .
\end{aligned}
$$

(3) $(\mu, \nu)=(n+1 / 2, m)$ and $(n, m) \in \mathbb{N}_{0} \times \mathbb{N}_{0}$.

$$
\begin{aligned}
& \int_{0}^{\pi / 2}(\log \sin \theta)^{2}(\sin \theta)^{2(n+1)}(\cos \theta)^{2 m+1} d \theta \\
& =\frac{2^{2 m-1} m !(2 n+2) !(m+n+2) !}{(n+1) !(2 m+2 n+4) !} \\
& \quad \cdot\left[4\left(O_{n+1}-O_{n+m+2}\right)^{2}+H_{m+1}^{(2)}\left(n+\frac{1}{2}\right)\right] .
\end{aligned}
$$

(4) $(\mu, \nu)=(n+1 / 2, m+1 / 2)$ and $(n, m) \in \mathbb{N}_{0} \times \mathbb{N}_{0}$.

$$
\begin{aligned}
& \int_{0}^{\pi / 2}(\log \sin \theta)^{2}(\sin \theta)^{2(n+1)}(\cos \theta)^{2(m+1)} d \theta \\
& =\frac{\pi(2 n+2) !(2 m+2) !}{2^{2 n+2 m+7}(n+1) !(m+1) !(n+m+2) !} \\
& \quad \cdot\left[\left(2 \log 2-2 O_{n+1}+H_{n+m+2}\right)^{2}+\frac{\pi^{2}}{3}-4 O_{n+1}^{(2)}+H_{n+m+2}^{(2)}\right]
\end{aligned}
$$

Differentiating both sides of Equation (3.3) with respect to $\mu$, we obtain 
(3.8)

$$
\begin{aligned}
& \int_{0}^{\pi / 2}(\log \sin \theta)^{3}(\sin \theta)^{2 \mu+1}(\cos \theta)^{2 \nu+1} d \theta \\
& =\frac{1}{16} \frac{\Gamma(\mu+1) \Gamma(\nu+1)}{\Gamma(\mu+\nu+2)}\left[(\psi(\mu+1)-\psi(\mu+\nu+2))^{3}\right. \\
& \quad+3(\psi(\mu+1)-\psi(\mu+\nu+2))\left(\psi^{\prime}(\mu+1)-\psi^{\prime}(\mu+\nu+2)\right) \\
& \left.\quad+\psi^{(2)}(\mu+1)-\psi^{(2)}(\mu+\nu+2)\right] \quad(\Re(\mu)>-1 ; \Re(\nu)>-1) .
\end{aligned}
$$

Here we give four special cases of Equation (3.8).

(1) $(\mu, \nu)=(n, m) \in \mathbb{N}_{0} \times \mathbb{N}_{0}$.

$$
\begin{aligned}
& \int_{0}^{\pi / 2}(\log \sin \theta)^{3}(\sin \theta)^{2 n+1}(\cos \theta)^{2 m+1} d \theta \\
& =\frac{n ! m !}{16(n+m+1) !} \\
& \quad \cdot\left[\left(H_{n}-H_{n+m+1}\right)^{3}+3\left(H_{n}-H_{n+m+1}\right) H_{m+1}^{(2)}(n)-2 H_{m+1}^{(3)}(n)\right] . \\
& \text { (2) }(\mu, \nu)=(n, m+1 / 2) \text { and }(n, m) \in \mathbb{N}_{0} \times \mathbb{N}_{0} .
\end{aligned}
$$

$$
\begin{aligned}
& \int_{0}^{\pi / 2}(\log \sin \theta)^{3}(\sin \theta)^{2 n+1}(\cos \theta)^{2(m+1)} d \theta \\
& =\frac{2^{2 n-2} n !(2 m+2) !(n+m+2) !}{(m+1) !(2 n+2 m+4) !}\left[\left(2 \log 2+H_{n}-2 O_{n+m+2}\right)^{3}\right. \\
& \quad+3\left(2 \log 2+H_{n}-2 O_{n+m+2}\right)\left(-\frac{\pi^{2}}{3}+4 O_{n+m+2}^{(2)}-H_{n}^{(2)}\right) \\
& \left.\quad+2\left(6 \zeta(3)+H_{n}^{(3)}+8 O_{n+m+2}^{(3)}\right)\right] .
\end{aligned}
$$

(3) $(\mu, \nu)=(n+1 / 2, m)$ and $(n, m) \in \mathbb{N}_{0} \times \mathbb{N}_{0}$.

$$
\begin{aligned}
& \int_{0}^{\pi / 2}(\log \sin \theta)^{3}(\sin \theta)^{2(n+1)}(\cos \theta)^{2 m+1} d \theta \\
& =\frac{2^{2 m-2} m !(2 n+2) !(m+n+2) !}{(n+1) !(2 m+2 n+4) !}\left[8\left(O_{n+1}-O_{n+m+2}\right)^{3}\right. \\
& \left.\quad+6\left(O_{n+1}-O_{n+m+2}\right) H_{m+1}^{(2)}\left(n+\frac{1}{2}\right)-2 H_{m+1}^{(3)}\left(n+\frac{1}{2}\right)\right] .
\end{aligned}
$$


(4) $(\mu, \nu)=(n+1 / 2, m+1 / 2)$ and $(n, m) \in \mathbb{N}_{0} \times \mathbb{N}_{0}$.

(3.12)

$$
\begin{aligned}
\int_{0}^{\pi / 2} & (\log \sin \theta)^{3}(\sin \theta)^{2(n+1)}(\cos \theta)^{2(m+1)} d \theta \\
= & \frac{\pi(2 n+2) !(2 m+2) !}{2^{2 n+2 m+8}(n+1) !(m+1) !(n+m+2) !} \\
& \cdot\left[\left(-2 \log 2+2 O_{n+1}-H_{n+m+2}\right)^{3}\right. \\
& +3\left(-2 \log 2+2 O_{n+1}-H_{n+m+2}\right)\left(\frac{\pi^{2}}{3}-4 O_{n+1}^{(2)}+H_{n+m+2}^{(2)}\right) \\
& \left.+2\left(-6 \zeta(3)-H_{n+m+2}^{(3)}+8 O_{n+1}^{(3)}\right)\right] .
\end{aligned}
$$

The case of $(1.5)$ when $p=1=q$ gives

$$
\begin{array}{r}
\int_{0}^{\pi / 2}(\log \sin \theta)(\log \cos \theta)(\sin \theta)^{2 \mu+1}(\cos \theta)^{2 \nu+1} d \theta \\
=\frac{1}{8} \frac{\Gamma(\mu+1) \Gamma(\nu+1)}{\Gamma(\mu+\nu+2)} \cdot[(\psi(\mu+1)-\psi(\mu+\nu+2))(\psi(\nu+1) \\
\left.-\psi(\mu+\nu+2))-\psi^{\prime}(\mu+\nu+2)\right] \\
(\Re(\mu)>-1 ; \Re(\nu)>-1) .
\end{array}
$$

Here we give four special cases of Equation (3.13).

(1) $(\mu, \nu)=(n, m) \in \mathbb{N}_{0} \times \mathbb{N}_{0}$.

$$
\begin{aligned}
& \int_{0}^{\pi / 2}(\log \sin \theta)(\log \cos \theta)(\sin \theta)^{2 n+1}(\cos \theta)^{2 m+1} d \theta \\
& =\frac{n ! m !}{8(n+m+1) !}\left[\left(H_{n}-H_{n+m+1}\right)\left(H_{m}-H_{n+m+1}\right)+H_{n+m+1}^{(2)}-\frac{\pi^{2}}{6}\right] . \\
& (2)(\mu, \nu)=(n, m+1 / 2) \text { and }(n, m) \in \mathbb{N}_{0} \times \mathbb{N}_{0} . \\
& (3.15) \\
& \int_{0}^{\pi / 2}(\log \sin \theta)(\log \cos \theta)(\sin \theta)^{2 n+1}(\cos \theta)^{2(m+1)} d \theta \\
& =\frac{2^{2 n-1} n !(2 m+2) !(n+m+2) !}{(m+1) !(2 n+2 m+4) !} \\
& \quad \cdot\left[2\left(O_{m+1}-O_{n+m+2}\right)\left(2 \log 2+H_{n}-2 O_{n+m+2}\right)+4 O_{n+m+2}^{(2)}-\frac{\pi^{2}}{2}\right] .
\end{aligned}
$$


(3) $(\mu, \nu)=(n+1 / 2, m)$ and $(n, m) \in \mathbb{N}_{0} \times \mathbb{N}_{0}$.

$$
\begin{aligned}
& \int_{0}^{\pi / 2}(\log \sin \theta)(\log \cos \theta)(\sin \theta)^{2(n+1)}(\cos \theta)^{2 m+1} d \theta \\
& =\frac{2^{2 m-1} m !(2 n+2) !(n+m+2) !}{(n+1) !(2 n+2 m+4) !} \\
& \quad \cdot\left[2\left(O_{n+1}-O_{n+m+2}\right)\left(2 \log 2+H_{m}-2 O_{n+m+2}\right)+4 O_{n+m+2}^{(2)}-\frac{\pi^{2}}{2}\right] .
\end{aligned}
$$

(4) $(\mu, \nu)=(n+1 / 2, m+1 / 2)$ and $(n, m) \in \mathbb{N}_{0} \times \mathbb{N}_{0}$.

$$
\begin{aligned}
& \int_{0}^{\pi / 2}(\log \sin \theta)(\log \cos \theta)(\sin \theta)^{2(n+1)}(\cos \theta)^{2(m+1)} d \theta \\
& =\frac{\pi(2 n+2) !(2 m+2) !}{2^{2 n+2 m+7}(n+1) !(m+1) !(n+m+2) !}\left[H_{n+m+2}^{(2)}-\frac{\pi^{2}}{6}\right. \\
& \left.+\left(-2 \log 2+2 O_{n+1}-H_{n+m+2}\right)\left(-2 \log 2+2 O_{m+1}-H_{n+m+2}\right)\right] .
\end{aligned}
$$

\section{Concluding remarks}

In view of Equation (1.1), it is easy to see the following symmetric relation with respect to the variables $\alpha$ and $\beta$ :

$$
B(\alpha, \beta)=B(\beta, \alpha) .
$$

Since we begin by giving a partial differentiation to the Equation (1.3), which preserves the symmetry with respect to the variables $\mu$ and $\nu$, if we interchange $\sin \theta \leftrightarrow \cos \theta$ and $\mu \leftrightarrow \nu$ in each of those integrals given in Section 3, we can obtain formulas each of whose evaluations is the same as in the corresponding results in Section 3. For example, we present only one formula corresponding to Equation (3.4):

$$
\begin{aligned}
\int_{0}^{\pi / 2} & (\log \cos \theta)^{2}(\sin \theta)^{2 n+1}(\cos \theta)^{2 m+1} d \theta \\
\quad= & \frac{n ! m !}{8(n+m+1) !}\left[\left(H_{n}-H_{n+m+1}\right)^{2}+H_{m+1}^{(2)}(n)\right] \quad\left(n, m \in \mathbb{N}_{0}\right) .
\end{aligned}
$$

Here we showed how we can obtain some interesting and (potentially) useful integrals by using a well known function. We leave the integrals involved in more complicated integrands to the interested reader. 


\section{References}

[1] V. S. Adamchik and H. M. Srivastava, Some series of the Zeta and related functions, Analysis 18 (1998), 131-144.

[2] N. Batir, Integral representations of some series involving $\left(\begin{array}{c}2 k \\ k\end{array}\right)^{-1} k^{-n}$ and some related series, Appl. Math. Comput. 147 (2004), 645-667.

[3] M. G. Beumer, Some special integrals, Amer. Math. Monthly 68 (1961), 645647.

[4] J. Choi, Certain summation formulas involving harmonic numbers and generalized harmonic numbers, Appl. Math. Comput. 218 (2011), 734-740; doi: 10.1016/j.amc.2011.01.062.

[5] J. Choi, Finite summation formulas involving binomial coefficients, harmonic numbers and generalized harmonic numbers, J. Inequ. Appl. 2013, 2013:49. http://www . journalof inequalitiesandapplications.com/content/2013/ $1 / 49$

[6] J. Choi, Log-sine and log-cosine integrals, Honam Math. J. 35 (2013), no. 2, 137-146.

[7] J. Choi, Y. J. Cho, and H. M. Srivastava, Log-sine integrals involving series associated with the Zeta function and Polylogarithms, Math. Scand. 105 (2009), 199-217.

[8] J. Choi and H. M. Srivastava, Explicit evaluation of Euler and related sums, Ramanujan J. 10 (2005), 51-70.

[9] J. Choi and H. M. Srivastava, Some applications of the Gamma and Polygamma functions involving convolutions of the Rayleigh functions, multiple Euler sums and log-sine integrals, Math. Nachr. 282 (2009), 1709-1723.

[10] J. Choi and H. M. Srivastava, Explicit evaluations of some families of log-sine and log-cosine integrals, Integral Transforms Spec. Funct. 22 (2011), 767-783.

[11] J. Choi and H. M. Srivastava, Some summation formulas involving harmonic numbers and generalized harmonic numbers, Math. Computer Modelling 54 (2011), 2220-2234.

[12] M. W. Coffey, On some series representations of the Hurwitz zeta function, J. Comput. Appl. Math. 216 (2008), 297-305.

[13] L. Lewin, Polylogarithms and Associated Functions, Elsevier (North-Holland), New York, London and Amsterdam, 1981.

[14] Th. M. Rassias and H. M. Srivastava, Some classes of infinite series associated with the Riemann Zeta and Polygamma functions and generalized harmonic numbers, Appl. Math. Comput. 131 (2002), 593-605.

[15] L. C. Shen, Remarks on some integrals and series involving the Stirling numbers and $\zeta(n)$, Trans. Amer. Math. Soc. 347 (1995), 1391-1399.

[16] A. Sofo and H. M. Srivastava, Identities for the harmonic numbers and binomial coefficients, Ramanujan J. 25 (2011), 93-113.

[17] H. M. Srivastava and J. Choi, Series Associated with the Zeta and Related Functions, Kluwer Academic Publishers, Dordrecht, Boston and London, 2001.

[18] — Zeta and q-Zeta Functions and Associated Series and Integrals, Elsevier Science Publishers, Amsterdam, London, and New York, 2012. 
Department of Mathematics

Dongguk University

Gyeongju 780-714, Republic of Korea

E-mail: junesang@mail.dongguk.ac.kr 